\title{
Operaciones y destrezas implicadas en la toma de decisiones sobre una problemática energética, identificadas por maestros en formación inicial
}

\author{
Naira Díaz-Moreno ${ }^{1, a}$, Beatriz Crujeiras-Pérez ${ }^{2, b}$, Carolina Martín-Gámez ${ }^{3, c}$ y Alicia \\ Fernández-Oliveras ${ }^{4, \mathrm{~d}}$ \\ ${ }^{1}$ Departamento de Educación. Facultad de Ciencias de la Educación. Universidad de Almería. Almería. \\ España \\ ${ }^{2}$ Departamento de Didácticas Aplicadas. Facultad de Ciencias de la Educación (campus norte). Universidade \\ de Santiago de Compostela. Santiago de Compostela. España. \\ ${ }^{3}$ Departamento de Didáctica de la Matemática, las Ciencias Sociales y las Ciencias Experimentales. Facultad \\ de Ciencias de la Educación. Universidad de Málaga. Málaga. España \\ ${ }^{4}$ Departamento de Didáctica de las Ciencias Experimentales. Facultad de Ciencias de la Educación. \\ Universidad de Granada. Granada. España. \\ anairadia@ual.es,.bbeatri氵.crujeiras@usc.es,.cmartin@uma.es,dalilia@ugr.es
}

[Recibido: 13 Junio 2017. Revisado: 17 Noviembre 2017. Aceptado: 24 enero 2018]

\begin{abstract}
Resumen: Con este trabajo se pretende dar a conocer las concepciones preliminares de maestros en formación inicial acerca de las operaciones y destrezas necesarias para tomar decisiones sobre una problemática sociocientífica relacionada con la energía eléctrica. Los participantes son 72 estudiantes universitarios de Educación Primaria matriculados en una asignatura semestral de Didáctica de las Ciencias Experimentales. Los resultados revelan las dificultades de los maestros en formación para identificar destrezas requeridas en la toma de decisiones, tales como las comunicativas (e. j. puesta en común de resultados) o las de investigación (e. j. formulación de cuestiones que permitan obtener la información deseada). Por otro lado, en el proceso de toma de decisiones, son escasos los participantes que establecen alguna operación relacionada con la evaluación de las decisiones tomadas. Además, los participantes conciben el proceso para resolver el problema sociocientífico como cerrado, no sujeto a revisión. De los resultados se extrae la necesidad de abordar estas cuestiones en conjunto con la Naturaleza de la Ciencia, con el propósito de mejorar las concepciones y desempeños de los participantes en los procesos de toma de decisiones.
\end{abstract}

Palabras clave: Controversias Sociocientíficas; Toma de Decisiones; Naturaleza de la Ciencia; Formación del Profesorado; Energía Eléctrica.

Operations and skills involved in decision-making identified by pre-service teachers

Abtsract: This paper seeks to examine pre-service teachers' preliminary conceptions about the operations and skills involved in decision-making processes, in particular those related to a socioscientific issue about electrical energy. The participants are 72 pre-service Primary Teachers enrolled in a semester course of Science Education. The data collected include the individual participants' responses to two written open questions. The results reveal the difficulties of pre-service teachers to identify the skills involved in decision-making, such as those associated to both communicative aspects (e.g. persuasion) and investigative ones (e.g. data analysis). Regarding the operations, there are few participants who propose operations related to the evaluation of the decisions made. Moreover, participants conceive a closed, not subject to revision, process for solving the socioscientific issue. In light of the results, there is a need of addressing decision-making processes together with Nature of Science aspects in order to improve participants' conceptions and performances about decision-making processes.

Keywords: Socioscientific Issues; Decision-Making; Nature of Science; Teacher Training; Electrical Energy.

Para citar este artículo: Díaz-Moreno, N., Crujeiras-Pérez, B., Martín-Gámez, C. y Fernández-Oliveras, A. (2018) Operaciones y destrezas implicadas en la toma de decisiones sobre una problemática energética, identificadas por maestros en formación inicial. Revista Eureka sobre Enseñanza y Divulgación de las Ciencias 15(2), 2601. doi: 10.25267/Rev_Eureka_ensen_divulg_cienc.2018.v15.i2.2601 


\section{Introducción}

Este trabajo forma parte de un proyecto de investigación más amplio sobre la introducción de la Naturaleza de la Ciencia (NdC) en la formación inicial del profesorado, con la finalidad de mejorar la educación científica, siendo clave para ello la formación que se le da al profesorado (Acevedo-Díaz y García-Carmona, 2016). El estudio de la NdC ha sido una meta importante en la Enseñanza de las Ciencias en las últimas décadas y continúa siéndolo hoy en día (Erduran y Dagher, 2014; Lederman y Lederman, 2014) considerándose además de especial relevancia para alcanzar la alfabetización científica (Comisión Europea, 2012; OCDE, 2006, 2013), ya que es esencial preparar futuros científicos, miembros cultos de la sociedad y ciudadanos informados (Taber, 2017). El conocimiento científico debe ser parte esencial de la cultura personal que nos permite interpretar la realidad con racionalidad y libertad así como disponer de argumentos para tomar decisiones (Castaño, Cuello, Gutiérrez, Rivero, Sampedro y Solís, 2006). El sistema educativo, por tanto, debe proporcionar la formación necesaria para enfrentarse a esos asuntos relacionados con la ciencia y estar en condiciones de adoptar decisiones informadas sobre ellos. En consecuencia, la finalidad última de la educación científica será la alfabetización científica no reduciéndola a la finalidad propedéutica de formar nuevos científicos/as, sino de convertir la educación científica en parte esencial de la educación general de todas las personas, una ciencia escolar para todos y todas (Pedrinaci, Caamaño, Cañal y de Pro, 2012).

En este artículo, examinamos el proceso de toma de decisiones en la formación inicial del profesorado como una herramienta para alcanzar la alfabetización científica. Para ello utilizamos el contexto de una controversia sociocientífica, asumiendo la visión II de Roberts (2007) de la alfabetización científica y siempre dentro de una perspectiva de investigación holística de la NdC. Lederman y Lederman (2014) consideran que por sí misma la comprensión de la $\mathrm{NdC}$ no hace que una persona esté científicamente alfabetizada. Así, los individuos científicamente alfabetizados deben no sólo conocer aspectos relacionados con la $\mathrm{NdC}$ sino además tener una comprensión funcional acerca del contenido de la ciencia, saber cómo se desarrolló ese contenido (capacidad de hacer y saber hacer sobre las prácticas de investigación) además de la capacidad de tomar decisiones informadas sobre cuestiones científicas personales y sociales. Teniendo en cuenta esta perspectiva, las cuestiones o controversias sociocientíficas (CSC) son un contexto idóneo para que los estudiantes se impliquen en dichas cuestiones complejas, así como para el desarrollo de los conocimientos y procesos que contribuyen a lograr la alfabetización científica (Sadler, 2009; Zeidler, Applebaum y Sadler, 2011). Además, es necesario señalar que las CSC se asocian frecuentemente con una dimensión polémica, motivada por las diferencias entre el conocimiento de contenido y la $\mathrm{NdC}$, como las percepciones de riesgo, la interpretación empírica de datos y el impacto social de la ciencia y la tecnología (Levinson, 2006). El razonamiento involucrado en la discusión de problemáticas controvertidas requiere argumentar a favor o en contra de diferentes posiciones evitando el sesgo de confirmación (Kuhn, 1991). Estas operaciones, junto con otras, son imprescindibles en el proceso de toma de decisiones. Por tanto, el objetivo principal de esta investigación consiste en analizar los procesos y destrezas implicadas en la toma de decisiones que un grupo de futuros maestros de Educación Primaria identifican como necesarias para resolver un dilema sociocientífico sobre la energía eléctrica. Este objetivo de investigación se concreta a través de las siguientes preguntas: 1) ¿Qué operaciones señalan como necesarias los futuros maestros para tomar una decisión sobre la subida del precio de la energía en su comunidad de vecinos y de qué forma las representan? y 2) ¿Qué destrezas identifican como necesarias los futuros maestros para la ejecución de las operaciones anteriores? 


\section{Marco teórico}

\section{Las CSC como contexto para la toma de decisiones}

Uno de los principales objetivos de la enseñanza de las ciencias es preparar a la ciudadanía, no para ser experta en ciencias, sino para tomar parte en las decisiones que afectan a su salud, a su alimentación, al uso adecuado de nuevos materiales y tecnologías o al uso de la energía, entre otros (OECD, 2013).

Por otra parte, el concepto de energía es uno de los componentes clave en el currículo internacional de la enseñanza de las ciencias. No obstante, este conocimiento sigue siendo enseñado en muchas aulas desde una perspectiva puramente conceptual, a pesar de que cada vez es más explícita la necesidad de abordarlo como una problemática sociocientífica aplicándola al contexto de la Educación ciudadana y de la Educación para el Desarrollo Sostenible. En este sentido se pretende que los estudiantes se conviertan en ciudadanos científicamente alfabetizados, o en palabras de Feinstein (2011) en "competent outsiders", capaces de tomar decisiones fundamentadas acerca de la energía (Sakscheswki, Eggert, Schneider y Bogëholz, 2014).

Cuando hablamos de CSC nos referimos a aquellos problemas auténticos que surgen de manera frecuente en nuestra sociedad con implicaciones científicas y/o tecnológicas (Ariza, Abril, Quesada y García, 2014; Duschl, 1995). Son problemas relacionados con ciencia y sociedad (Kolstø, 2001) que denotan la compleja relación que existe entre ambas, y que normalmente aparecen cuando existe una discrepancia por intereses de diversa naturaleza entre los diversos actores, fuerzas sociales o entidades civiles que participan en ella (investigadores, científicos, opinión pública, administración, empresas privadas que financian los estudios, etc.) (Díaz-Moreno y Jiménez-Liso, 2012). Es decir, estas cuestiones además de ser socialmente controvertidas incorporan otras disciplinas e intereses (políticos, económicos, etc.) e implican, a su vez, la evaluación de aspectos relacionados con la cuestión moral y la ética (Evagorou, Jimenez-Aleixandre y Osborne, 2012; Zeidler y Nichols, 2009). Son, por tanto, asuntos sociales controvertidos con vínculos conceptuales y/o procedimentales con la ciencia, que se caracterizan por ser complejos, abiertos y carecer de soluciones simples y directas (Sadler, 2004; Sadler, Barab y Scott, 2007). Las posibles soluciones de estas cuestiones pueden ser informadas por principios científicos, teorías y datos, pero además están influenciadas por distintos factores sociales e implican connotaciones éticas y morales (Levinson, 2006; Ratcliffe y Grace, 2003; Sadler, 2011). Como decimos, en algunos de estos problemas no existen respuestas definitivas, y cualquiera que sea la postura que el individuo o la sociedad tenga ante ellos, el debate no le va a ser ajeno, ya que su importancia va a ir en aumento a medida que prosiguen los avances de la ciencia y los problemas derivados de su aplicación (España y Prieto, 2009).

En este trabajo planteamos el contexto de un dilema sociocientífico concreto sobre la energía eléctrica para trabajar la toma decisiones y el desarrollo de destrezas, que exponemos más adelante. Tomamos como base la importancia que tiene preparar a los estudiantes para tomar decisiones fundamentadas e informadas (NRC, 1996), puesto que a diario nos enfrentamos a situaciones relacionadas con la ciencia y tecnología en el contexto de problemas de actualidad, cuyas repercusiones no sólo nos afectan individualmente sino también a nivel global.

\section{La toma de decisiones como herramienta para promover el desarrollo de destrezas}

Como hemos señalado en la sección anterior, uno de los propósitos fundamentales en la Didáctica de las Ciencias consiste en enseñar a los estudiantes a pensar de forma crítica y a ser ciudadanos participativos, capaces de tomar decisiones bien fundamentadas (Gresch, 
Hasselhorn y Bögeholz, 2013). Además, la toma de decisiones es uno de los aspectos a desarrollar para alcanzar la alfabetización científica (Kifshe, 2012) ya que ésta permite el desempeño de un papel más activo y efectivo en la sociedad, así como incrementar la conciencia social sobre la dimensión de las decisiones y elecciones tomadas (Ratcliffe y Grace, 2003). Por ello, puede considerarse que la toma de decisiones es un proceso de aprendizaje que implica que los estudiantes propongan y discutan sobre un punto de vista $\mathrm{O}$ posicionamiento en una situación determinada que implica la selección entre distintas alternativas (Kortland, 1996).

En la literatura existen distintos marcos de análisis para los procesos de toma de decisiones y las operaciones implicadas en los mismos. Un ejemplo es el marco propuesto por Heath, White, Berlin y Park (1987), y que se ha tenido en cuenta para elaborar una de las herramientas de análisis de los datos en este artículo, tal y como se detalla en la siguiente sección. Según estos autores el proceso de toma de decisiones comprende de cinco operaciones implicadas: 1) identificar el problema; 2) recopilar información relevante para resolver el problema; 3) elaborar posibles soluciones; 4) evaluar las soluciones; y 5) seleccionar propuestas.

Por otro lado, Ractliffe (1997) amplía esta propuesta a una guía de seis etapas dado que propone promover la identificación de criterios relevantes y la discusión sobre los beneficios e inconvenientes de una decisión: 1) Opciones, que implica la operación de identificar las posibles alternativas; 2) Criterios, que incluye la operación de establecer criterios adecuados para comparar las alternativas; 3) Información, que engloba la operación de aclarar la información conocida sobre las posibles alternativas teniendo en cuenta los criterios establecidos, así como el conocimiento y las pruebas científicas; 4) Sondeo, que implica analizar las ventajas e inconvenientes de cada alternativa en base a los criterios establecidos; 5) Selección, que comprende el escoger una de las alternativas posibles en base al análisis realizado en la etapa anterior; y 6) Revisión, que requiere evaluar todo el proceso de toma de decisiones para identificar posibles mejoras.

Esta propuesta se complementa con la realizada por Jiménez-Aleixandre y Pereiro (2002) que señalan diferentes dimensiones dentro de la toma de decisiones, entre ellas: 1) el uso del conocimiento relevante para comprender y tomar decisiones sobre el problema, 2) el procesado crítico de fuentes de información y 3) la elaboración de criterios para evaluar soluciones posibles al problema. Otros autores como Lee y Grace (2012) diseñan y utilizan un marco que comprende 3 grandes fases divididas en ocho etapas, de las cuales las seis primeras son similares a las propuestas por Ratcliffe (1997), mientras que las dos últimas están encaminadas a la puesta en común de las decisiones justificadas, con alumnado tanto del mismo entorno como de otros diferentes, con el propósito de revisar las decisiones tomadas.

En definitiva, todos estos planteamientos requieren el manejo de una serie de destrezas y operaciones a desarrollar durante la etapa educativa como son la elaboración de argumentos consistentes y basados en pruebas (Kifshe, 2012) o la evaluación crítica de dichos argumentos (Kolstø et al., 2006). Además, también es necesario tener conocimiento sobre otros aspectos, como la comprensión de que las cuestiones reales tienen más de una respuesta y que en este tipo de cuestiones no se busca la respuesta correcta, sino la mejor dentro de un conjunto de restricciones (Zoller, 1982). También es importante la identificación y comprensión del conocimiento científico implicado en la cuestión objeto de estudio (Bingle y Gaskell, 1994), la consideración de alternativas o la evaluación de consecuencias morales (Ratcliffe, 1997).

De todo ello se deriva, por tanto, que la toma de decisiones es un proceso complejo que comprende numerosas etapas, lo cual supone dificultades para el alumnado. Entre las dificultades señaladas en la literatura se encuentra: 1) la evaluación de la información (JiménezAleixandre y Pereiro-Muñoz, 2002), 2) evaluación de los argumentos y de los criterios 
utilizados para tomar una decisión fundamentada (Kolstø et al., 2006), 3) así como de la capacidad para reflexionar y regular los propios procesos de toma de decisiones (Papadouris, 2012). Además de dificultades relacionadas con operaciones cognitivas, cuando la toma de decisiones se enmarca en cuestiones sociocientíficas, como en este estudio, se identifican también dificultades morales como la aceptación de pruebas que contradicen los propios pensamientos (Zeidler, 1997) ya que los valores personales tienen un papel importante en estos procesos de toma de decisiones, como señalan Bell y Lederman (2003) o Kolstø et al. (2006), entre otros autores.

Para solventar las dificultades es necesario proporcionar apoyo docente, lo que implica que el profesorado debe tener herramientas y recursos para formar a los estudiantes en estos procesos, a la vez que se trabajan los contenidos científicos y se desarrollan las competencias en el aula. En este sentido, para que los apoyos proporcionados sean adecuados, es necesario formar al profesorado en estas cuestiones. Por ello, en este trabajo analizamos las operaciones y destrezas identificadas como relevantes, por los maestros de Educación Primaria en formación inicial, para tomar una decisión sobre una cuestión sociocientífica enmarcada en el consumo de energía eléctrica.

\section{Metodología}

Los participantes son 72 profesores de Educación Primaria en formación inicial. Concretamente, se trata de estudiantes de tercer curso del Grado de Maestro en Educación Primaria de la Universidad de Granada, que estaban cursando la primera asignatura de Didáctica de las Ciencias Experimentales de su titulación durante el primer semestre del curso académico 2016-2017.

Los datos se recogieron en la primera sesión (90 minutos) de la mencionada asignatura e incluyen notas de campo así como las respuestas escritas de los participantes a las dos preguntas abiertas siguientes:

1. La compañía eléctrica de tu comunidad de vecinos anuncia que va subir el precio del Kwh un $100 \%$ y el problema se plantea en una reunión. Indica a modo de esquema las operaciones que se deberían realizar para tomar una decisión al respecto (el esquema debe mostrar la secuenciación de las operaciones y las posibles relaciones entre ellas).

2. ¿Qué destrezas crees que se ponen en juego en las operaciones que has planteado?

La metodología se basa en el análisis cualitativo del contenido (Schreirer, 2012), con el fin de describir sistemáticamente el significado del material cualitativo recopilado, en este caso las respuestas escritas del profesorado en formación, clasificándolo mediante las categorías de un marco de codificación. Considerando este marco, cada una de las autoras de este trabajo realizó una primera revisión de los datos. A continuación se elaboró una codificación provisional por pares comparando los resultados obtenidos, obteniendo un $90 \%$ de coincidencia en el análisis. Los resultados no coincidentes se recodificaron hasta llegar al 100\% de acuerdo entre las cuatro integrantes.

Dado los objetivos planteados en este trabajo y la sencillez de la propuesta de Heath, White, Berlin y Park (1987), decidimos utilizarla como herramienta para el análisis de las respuestas a la primera pregunta (tabla 1). A partir de los esquemas realizados por los participantes, estudiamos también la coherencia en el orden de las operaciones planteadas y si su conexión da lugar a una secuencia lineal o recursiva, apoyándonos en el trabajo de Escrivà-Colomar y Rivero-García (2017). 
Tabla 1. Sistema de análisis de las operaciones implicadas en la toma de decisiones (Heath, White, Berlin y Park, 1987).

\begin{tabular}{|l|l|}
\hline Operación & Descripción \\
\hline 1. Identificar del problema & Señalar la naturaleza de la situación que supone una dificultad. \\
\hline $\begin{array}{l}\text { 2. Recopilar información relevante } \\
\text { para resolver el problema }\end{array}$ & Recoger datos de utilidad para solventar la dificultad señalada. \\
\hline 3. Elaborar posibles soluciones & $\begin{array}{l}\text { Diseñar estrategias que potencialmente permitan solventar la dificultad } \\
\text { señalada. }\end{array}$ \\
\hline 4. Evaluar las soluciones & Valorar comparativamente las distintas estrategias diseñadas. \\
\hline 5. Seleccionar propuestas & Escoger una de las estrategias consideradas. \\
\hline
\end{tabular}

Por otro lado, las destrezas indicadas en las respuestas a la segunda pregunta se analizaron empleando el sistema de categorías que se muestra en la tabla 2. Estas categorías se agrupan en cuatro tipos, siendo tres de ellos los ámbitos que de Pro (2013) utiliza para clasificar procedimientos (destrezas básicas, destrezas de investigación y destrezas comunicativas) y el cuarto tipo una categoría emergente relacionada con el contexto de la tarea (destrezas sociales).

Tabla 2. Sistema de análisis de las destrezas implicadas en la toma de decisiones.

\begin{tabular}{|c|c|c|}
\hline Destrezas & Descripción & Categoría \\
\hline Destrezas Básicas & $\begin{array}{l}\text { Son las propias de la obtención } \\
\text { de información, su tratamiento } \\
\text { y evaluación. }\end{array}$ & $\begin{array}{l}\text { DB1. Obtención, organización y evaluación la } \\
\text { información; DB2. Realización de operaciones } \\
\text { matemáticas; DB3. Comparación y selección de } \\
\text { distintas propuestas según su idoneidad. }\end{array}$ \\
\hline Destrezas Sociales & $\begin{array}{l}\text { Son las implicadas en la } \\
\text { relación entre individuos. }\end{array}$ & $\begin{array}{l}\text { DS1. Comunicación interpersonal; DS2. Búsqueda } \\
\text { de consenso entre las distintas opciones } \\
\text { propuestas. }\end{array}$ \\
\hline Destrezas de Investigación & $\begin{array}{l}\text { Son las asociadas a la } \\
\text { identificación y búsqueda de } \\
\text { soluciones a un problema. }\end{array}$ & $\begin{array}{l}\text { DI1. Identificación de hechos o situaciones } \\
\text { problemáticas; DI2. Formulación de cuestiones } \\
\text { que permita obtener la información deseada; DI3. } \\
\text { Emisión de hipótesis; DI4. Interpretación y } \\
\text { análisis de datos; DI5. Construcción de } \\
\text { explicaciones fundamentadas; DI6. Toma de } \\
\text { decisiones. }\end{array}$ \\
\hline Destrezas Comunicativas & $\begin{array}{l}\text { Son las relacionadas con la } \\
\text { exposición razonada de la } \\
\text { información. }\end{array}$ & $\begin{array}{l}\text { DC1. Oratoria; DC2. Argumentación; DC3. } \\
\text { Persuasión; DC4. Divulgación de medidas DC5. } \\
\text { Contraste de ideas en material audiovisual; DC6. } \\
\text { Puesta en común de los resultados. }\end{array}$ \\
\hline
\end{tabular}

\section{Resultados}

\section{a. Concepciones de los participantes sobre las operaciones involucradas en la toma de decisiones ante una problemática sociocientífica.}

De manera general, los datos muestran que el profesorado en formación inicial (PF) identifica un bajo número de operaciones a realizar ante la resolución de una problemática sociocientífica. Esto se pone de manifiesto dado que 47 de ellos consideran que se deberían ejecutar dos o tres operaciones en sus planteamientos, 13 propone la realización de cuatro y solamente uno de ellos recoge en sus respuestas las cinco operaciones que podríamos considerar deseables de llevar a cabo ante una toma de decisiones (Heath, White, Berlin y Park, 1987). 
En relación a las operaciones específicas que identifican, la tabla 3 muestra las operaciones consideradas en el análisis y el número de participantes que identifica cada una de ellas.

Tabla 3. Operaciones a realizar ante la toma de decisiones, frecuencias y ejemplos de respuesta.

\begin{tabular}{|l|c|l|}
\hline \multicolumn{1}{|c|}{ Operación } & Frecuencia & \multicolumn{1}{|c|}{ Ejemplo/s de respuesta } \\
\hline 1. Identificar del problema & 34 & $\begin{array}{l}\text { "Previamente se explica en la reunión de vecinos lo } \\
\text { sucedido" (PF.6) }\end{array}$ \\
\hline $\begin{array}{l}\text { 2. Recopilar información relevante } \\
\text { para resolver el problema }\end{array}$ & 23 & $\begin{array}{l}\text { "Buscar el porqué de ese aumento" (PF.11) } \\
\text { "Consultar con un experto en electricidad" (PF.22) }\end{array}$ \\
\hline 3. Elaborar posibles soluciones & 40 & $\begin{array}{l}\text { "Presentar soluciones" (PF.1) } \\
\text { "Buscar alternativas de suministros energéticos" (PF.54) }\end{array}$ \\
\hline 4. Evaluar las soluciones & 34 & $\begin{array}{l}\text { "Se estudiarán los pros y los contras de cada solución" } \\
\text { "PF.26) }\end{array}$ \\
\hline "Valorar aspectos positivos/negativos" (PF.34)
\end{tabular}

Los resultados evidencian cómo la operación a la que menos aluden es la recopilación de la información relevante, dado que sólo el 31,9\% de los participantes parecen considerar ésta como una de las operaciones fundamentales para adoptar una decisión. Este dato resulta llamativo si es contrastado con los resultados obtenidos relacionados con las operaciones que reciben más alusiones. Como se aprecia en la tabla 3, el 55,6\% de los participantes elaborarían soluciones a la problemática planteada pero para ello solo el 6,9\% recopilarían información en el momento inicial, para definir bien el problema, y con posterioridad, para elaborar las posibles soluciones (imagen 1); el 11,1\% participantes solo la recopilarían inicialmente; y el $13,9 \%$ solo realizarían dicha operación en algún momento del proceso con el fin de recabar datos que les permitan presentar las posibles soluciones (imagen 2).
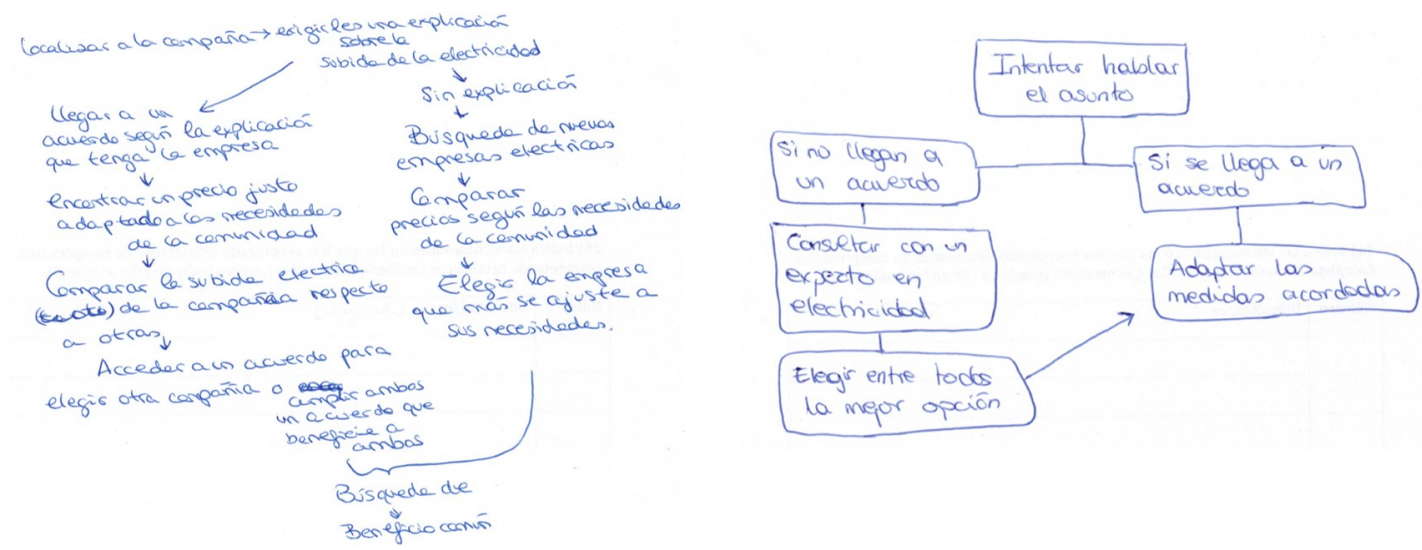

Imagen 1. Esquema de operaciones con recopilación Imagen 2. Esquema de operaciones con recopilación de de información inicial y durante el proceso (PF.12) información durante el proceso (PF.10)

Los 17 restantes no consideran necesaria recopilar información en ningún momento del proceso, y creen suficiente generar las posibles soluciones a partir de sus conocimientos previos y opiniones en la materia (imagen 3 ).

Los datos también muestran que el 22,2\% de los participantes no proponen ningún proceso para llegar a seleccionar una propuesta y por tanto, llegar a adoptar una decisión (tabla 3). Las respuestas del $77,8 \%$ que sí lo harían, se agrupan entre los que sugieren realizar una votación en la que la solución vendrá impuesta por la mayoría (29,2\%); los que plantean que la decisión sea tomada de manera unilateral por los representantes de la comunidad (8,3\%); y aquellos que consideran necesario buscar el acuerdo y el consenso colectivo entre los implicados (40,3\%). 
En relación a estos últimos, resulta llamativo observar como el 13,9\% no establece operación alguna para evaluar las posibles soluciones, y propone la búsqueda de consensos sin aportar datos o evidencias que permitan conformar argumentos sobre los que consensuar dichas soluciones (Imagen 4).

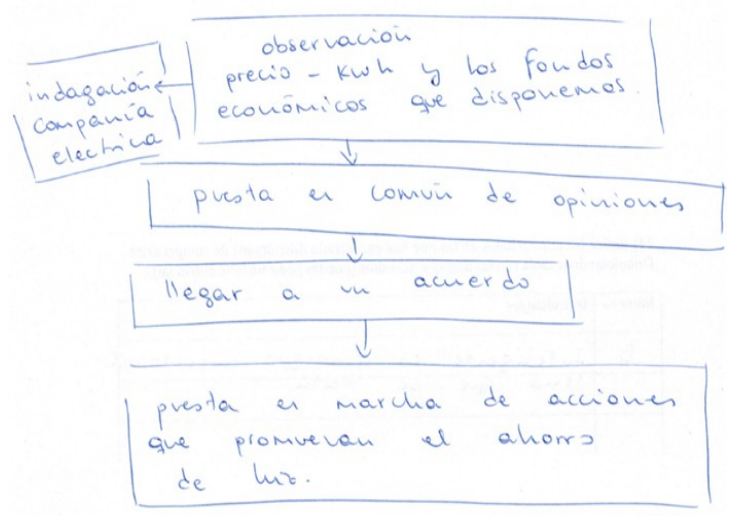

Imagen 3. Esquema de operaciones sin recopilación de información (PF.31)

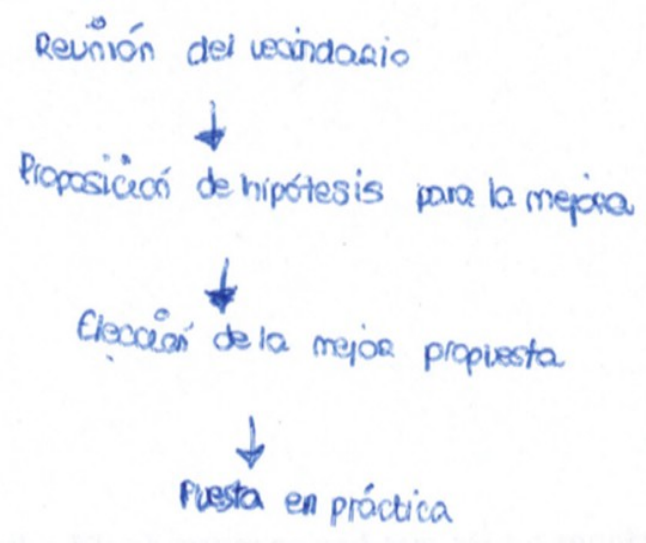

Imagen 4. Esquema de operaciones sin evaluación de soluciones (PF.14)

Además, se aprecia que un $27,8 \%$ de los participantes presentan esquemas con cierta falta de coherencia en el orden en el que proponen las operaciones a realizar ante la problemática. Muestra de esta inconsistencia es que en muchos de estos casos los participantes seleccionarían las propuestas y posteriormente recopilarían información relevante sobre la temática. También destacar que existe un número muy alto de participantes, en concreto el $87,5 \%$, que presenta un esquema de operaciones caracterizado por ser lineal y cerrado, es decir, constituido por una serie de pasos a seguir entre un inicio y un final completamente acabado (imagen 3 e imagen 4). Solo un 6,9\% propone esquemas en los que se contempla cierta recursividad en sus operaciones (imagen 5).

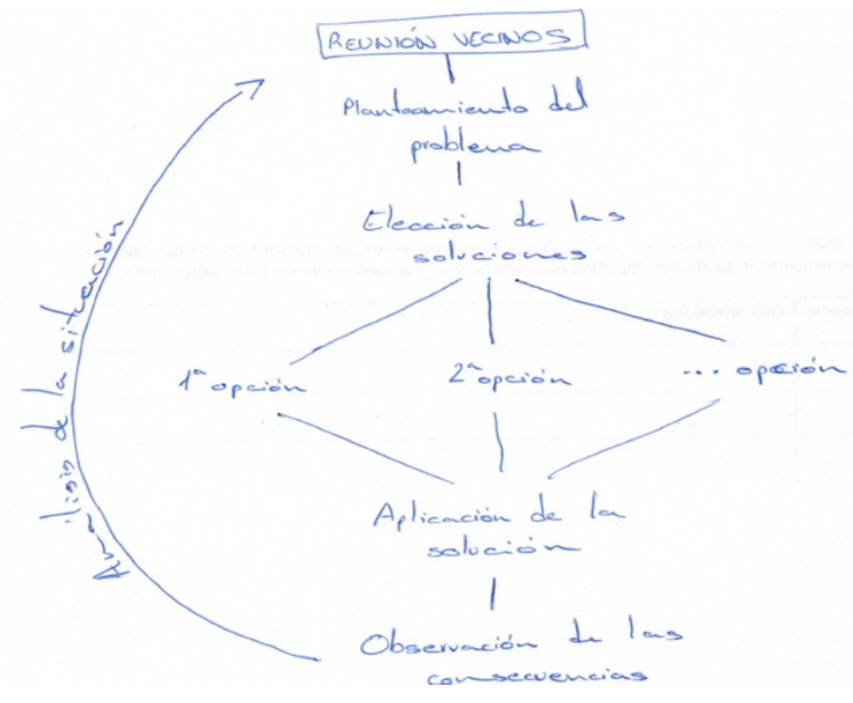

Imagen 5. Esquema de operaciones con recursividad (PF.28) 


\section{b. Concepciones de los participantes sobre las destrezas requeridas en la toma de decisiones ante una problemática sociocientífica.}

Los resultados ponen de manifiesto que los participantes identifican un bajo número de destrezas que deberían ponerse en juego para tomar decisiones ante una problemática sociocientífica. Este hecho se constata dado que son tres el número máximo de destrezas que son capaces de identificar de las 17 consideradas en el análisis y, en concreto, lo hacen un $18,1 \%$ de participantes. Además, el 22,2\% de participantes no identifica destreza alguna en su respuesta, el 25\% recoge solamente una y el 34,7\% menciona dos de ellas.

De manera más específica, en relación a las destrezas concretas que proponen, la tabla 4 recoge las categorías consideradas en el estudio y las frecuencias con que aparecen. También ha sido calculada la frecuencia relativa que expresa el porcentaje de respuesta de las cuatro grandes categorías de destrezas consideradas (básicas, sociales, de investigación y comunicativas) respecto al total de respuesta.

Como se observa en la tabla 4, las destrezas que menos atención reciben son las consideradas destrezas básicas, mientras que la más identificadas son las relacionadas con las destrezas de investigación. En relación a las primeras, el 22,2\% que las tratan lo hacen porque aluden a una sola de las categorías que conforman dicha destreza como, por ejemplo: "Organización de ideas"(PF. 1, Categoría DB1) o "Clasificación de ideas" (PF. 29, Categoría DB3).

Respecto a las destrezas de investigación, el 47,2\% las consideran, mostrando en sus respuestas una o dos de las categorías relacionadas. Concretamente, el 9,7\% lo hace mencionando dos de las categorías: "[...] encontrar varias soluciones posibles y alternativas al problema (buscar otras empresas; [...] y focalizar la atención en un problema y su solución (¿Cómo llego a un acuerdo que beneficie ambas partes?" (PF. 12, Categorías DI1 y DI5); y el 27,8\% restante únicamente menciona 1 de las categorías: "Habilidad de plantear hipótesis referidas a los planteamientos de cada vecino en el debate [...]" (PF.71, Categoría DI3). Además los datos muestran cómo las respuestas que apuntan hacia las destrezas de investigación se centran en destacar la categoría "toma de decisiones" (DI6), estando el resto de categorías que la conforman muy poco aludidas.

Tabla 4. Destrezas implicadas en la toma de decisiones, frecuencia y frecuencia relativa.

\begin{tabular}{|c|c|c|c|}
\hline Destrezas & Categorías & Frecuencia & $\begin{array}{l}\text { Frecuencia } \\
\text { relativa }\end{array}$ \\
\hline \multirow{3}{*}{$\begin{array}{l}\text { Destrezas } \\
\text { Básicas }\end{array}$} & DB1. Obtención, organización y evaluación de la información & 6 & \multirow{3}{*}{$14,95 \%$} \\
\hline & DB2. Realización de operaciones matemáticas & 1 & \\
\hline & $\begin{array}{l}\text { DB3. Comparación y selección de distintas propuestas según su } \\
\text { idoneidad }\end{array}$ & 9 & \\
\hline \multirow[b]{2}{*}{$\begin{array}{l}\text { Destrezas } \\
\text { Sociales }\end{array}$} & DS1. Comunicación interpersonal & 13 & \multirow[b]{2}{*}{$29,91 \%$} \\
\hline & $\begin{array}{l}\text { DS2. Búsqueda de consenso entre las distintas opciones } \\
\text { propuestas }\end{array}$ & 19 & \\
\hline \multirow{6}{*}{$\begin{array}{l}\text { Destrezas } \\
\text { de } \\
\text { Investigació } \\
\mathrm{n}\end{array}$} & DI1. Identificación de hechos o situaciones problemáticas & 5 & \multirow{6}{*}{$31,78 \%$} \\
\hline & $\begin{array}{l}\text { DI2. Formulación de cuestiones que les permitan obtener la } \\
\text { información deseada }\end{array}$ & 0 & \\
\hline & DI3. Emisión de hipótesis sobre las posibles medidas & 7 & \\
\hline & DI4. Interpretación y análisis de datos & 7 & \\
\hline & DI5. Construcción de explicaciones fundamentadas & 2 & \\
\hline & DI6. Toma de decisiones & 13 & \\
\hline \multirow{6}{*}{$\begin{array}{l}\text { Destrezas } \\
\text { Comunicati } \\
\text { vas }\end{array}$} & DC1. Oratoria & 14 & \multirow{6}{*}{$23,36 \%$} \\
\hline & DC2. Argumentación & 8 & \\
\hline & DC3. Persuasión & 2 & \\
\hline & DC4. Divulgación de medidas & 1 & \\
\hline & DC5. Contraste de ideas en material audiovisual & 0 & \\
\hline & DC6. Puesta en común de los resultados & 0 & \\
\hline
\end{tabular}


Por otro lado, en relación a las destrezas comunicativas, es posible comprobar cómo la categoría más mencionada es la oratoria $(19,4 \%$ ) y es casi la única junto con la argumentación (DC2) que identifican dentro de este tipo de destrezas (tabla 4). Hacer constar que solo el $1,4 \%$ de participantes que menciona alguna de las categorías que componen esta destreza, considera dos de ellas: "Habilidad de debatir; [...]; habilidad de dialogar" (PF. 37, Categorías DC1 y DC2); el resto solo considera: "[...] habilidades negociadoras y mediadoras [...]" (PF. 39, Categoría DC3); "El hecho de comunicarse para solventar los problemas, es decir, la capacidad de comunicación" (PF. 18, Categoría DC1).

Por último, las categorías relacionadas con las destrezas sociales son de las más aludidas $(44,4 \%)$ aunque, siguiendo la misma línea que en los casos anteriores, solo el 2,8\% de los participantes aluden a las dos categorías consideradas: "Aceptar otras opiniones que no son tuyas; saber escuchar; respetar a los demás" (PF. 19 Categorías, DS1 y DS2).

\section{Conclusiones e implicaciones formativas}

Los resultados obtenidos señalan que los maestros en formación inicial identifican un bajo número de operaciones involucradas en el proceso de toma de decisiones sobre la cuestión sociocientífica planteada en este trabajo. Concretamente, la mayoría de los participantes identifica la necesidad de elaborar posibles soluciones, pero sin recopilar información, seleccionarla y evaluarla.

Además, los datos muestran cierta incoherencia en el esquema de operaciones propuesto por un gran número de participantes. Este hecho se pone de manifiesto en dos sentidos: 1) orden inadecuado en las operaciones, situando algunas de ellas antes que otras que proporcionarían información y datos necesarios para realizar las primeras; 2) identificación insuficiente de las operaciones necesarias para tomar decisiones de forma rigurosa y fundamentada, elaborando soluciones sin recopilar información o seleccionado propuestas sin evaluarlas. En relación a esto último, tan solo una cuarta parte de los participantes establece alguna operación para evaluar las posibles soluciones propuestas, como vía para seleccionar una de ellas. El resto alude fundamentalmente a adoptar la solución avalada por la mayoría, o a un consenso que no implica evaluar la adecuación de las propuestas. Todo ello evidencia que este profesorado en formación inicial dispone de un conocimiento muy limitado de los elementos necesarios que conforman una propuesta fundamentada y, en definitiva, un buen argumento que permita adoptar una decisión. Este desconocimiento podría traducirse en un enfoque de enseñanzaaprendizaje que no potencia en sus futuros estudiantes el desarrollo de operaciones que les permitan crear argumentos sólidos y evaluar los de otros, en contra de lo que correspondería a una buena manera de promover la comprensión del conocimiento científico (Erduran, Dilek y Yakmaci-Guzel, 2006).

Por otro lado, los participantes conciben el proceso para resolver el problema sociocientífico como cerrado, no sujeto a revisión. Es decir, se muestra cómo este profesorado en formación inicial dispone de una visión cerrada y absolutista del proceso a seguir, sin considerar que la aparición de nuevos datos o interpretaciones pueden plantear revisiones en el proceso y, por tanto, cambios en la toma de decisiones. Este resultado podría estar relacionado con el poco conocimiento de los participantes sobre $\mathrm{NdC}$, tal y como señalan Lederman y Lederman (2014).

Los resultados también ponen de manifiesto las dificultades de los participantes para identificar las destrezas implicadas en todo el proceso de toma de decisiones, ya que casi la mitad de ellos, en concreto el $47,2 \%$, solo identifica una o ninguna de las destrezas consideradas dentro del sistema de análisis propuesto. Entre dichas destrezas, la más frecuente 
es la búsqueda de consenso entre las distintas opciones propuestas, mientras que otras como la formulación de cuestiones que permitan obtener la información deseada, o la puesta en común de resultados no son identificadas por los participantes. Esto refuerza la idea señalada por Ryder (2001) de que la comprensión de la $\mathrm{NdC}$, en el sentido de cómo funciona la ciencia y produce conocimiento fiable, es importante para que los estudiantes se involucren críticamente en cualquier problemática sociocientífica.

Además, la importancia de las concepciones identificadas radica en la influencia que éstas puedan tener sobre las futuras prácticas docentes de los maestros en formación (Porlán y Martín del Pozo, 2004), concretamente, en lo que se refiere al desarrollo de las operaciones y destrezas necesarias para la toma de decisiones en sus futuros alumnos.

En consecuencia, si uno de los objetivos de la educación científica es mejorar el aprendizaje, y en concreto, proporcionar más oportunidades para que los estudiantes aprendan a construir argumentos sólidos, es necesario entonces que desde los programas formativos del profesorado de ciencias se promueva la reflexión y el razonamiento sobre la importancia de fomentar el uso de las operaciones involucradas para ello, y se trabaje el cómo hacerlo en el aula. Además, a raíz de los resultados de este estudio, y dada la importancia del conocimiento sobre NdC en el profesorado (Acevedo-Díaz y García-Carmona, 2016), sugerimos la necesidad de profundizar más en este conocimiento. Por ello, se considera necesario que desde su formación inicial se trabajen actividades sobre CSC de ámbito local en las que se deba adoptar una decisión, dado que esto les permitirá analizar la existencia de diferentes perspectivas, y diferentes caminos para la toma de decisiones (España y Prieto, 2009). El enfoque desde su formación no solo debe incluir el análisis sobre las posturas de los agentes implicados en las CSC, sino también la creación de argumentos basados en pruebas de cada uno de ellos, la confrontación de los mismos, así como, una reflexión sobre las operaciones llevadas a cabo. También, se propone la realización de una evaluación del proceso en forma de mapa, en el cuál se reflejen las destrezas puestas de manifiesto y las que se podrían haber usado para mejorar la toma de decisión adoptada.

Finalmente, se considera que este trabajo, cuyo propósito es conocer qué operaciones y destrezas utiliza el profesorado de Primaria en formación inicial ante una problemática sociocientífica, representa el punto de partida de un estudio más amplio acerca de la introducción de la $\mathrm{NdC}$ en la formación inicial de maestros. Para ello, y teniendo en cuenta las implicaciones formativas que emergen de este trabajo, se ha elaborado e implementado una secuencia de actividades encaminada a tal fin, y con la que también se trata de examinar el efecto de la misma en la evolución de las concepciones y desempeños de los maestros en formación sobre dichas cuestiones. Se pretende estudiar y analizar esta evolución no solo desde la perspectiva presentada en este trabajo, si no también considerando la calidad en cuanto a las operaciones identificadas y las destrezas necesarias para implementarlas.

\section{Referencias}

Acevedo-Díaz, J. A. y García-Carmona A. (2016) Algo antiguo, algo nuevo, algo prestado. Tendencias sobre la naturaleza de la ciencia en la educación secundaria. Revista Eureka sobre Enseñanza y Divulgación de las Ciencias, 13(1), 3-19.

Ariza, M. R., Abril, A.M., Quesada, A. y García, FJ (2014) Conectar el aprendizaje por investigación con controversias socio-científicas. Contribuciones del proyecto europeo PARRISE. Comunicación presentada en XXVI en Encuentros en Didáctica de las Ciencias Experimentales. Huelva. 
Bell, R. L. y Lederman, N. G. (2003) Understandings of the Nature of Science and Decision Making on Science and Technology Based Issues. Science Education, 87, 352-377.

Bingle, W. H., y Gaskell, P. J. (1994) Scientific literacy for decision making and the social construction of scientific knowledge. Science Education, 78(2), 185-201.

Castaño, E., Cuello, A., Gutiérrez, N., Rivero, A, Sampedro, C. y Solís, E. (2006) Educación y cultura científica. Sevilla: Junta de Andalucía. Consejería de Educación.

Comisión Eureopea (2012)/Eurydice (2012) Developing Key Competences at School in Europe: Challenges and Opportunities for Policy - 2011/12. Eurydice Report. Luxembourg: Publications Office of the European Union.

Díaz-Moreno, N. y Jiménez-Liso, M.R. (2012). Las controversias sociocientíficas: temáticas e importancia para la educación científica. Revista Eureka sobre Enseñanza y Divulgación de las Ciencias, 9(1), 54-70.

Duschl R. A. (1995) Más allá del conocimiento: los desafíos epistemológicos y sociales de la enseñanza mediante el cambio conceptual. Enseñanza de las Ciencias, 13(1), 3-14.

Erduran, S. y Dagher, Z. (2014) Reconceptualizing the Nature of Science for Science Education. Dordrecht: Springer.

Erduran S., Dilek A., y Yakmaci-Guzel, B. (2006) Learning to teach argumentation: Case studies of pre-service secondary science teachers. Eurasia Journal of Mathematics, Science and Technology Education, 2 (2), 1-14.

Escrivà-Colomar, I., y Rivero, A. (2017).Progresión de las ideas de los futuros maestros sobre la construcción del conocimiento científico a través de mapas generados en una secuencia de actividades. Revista Eureka sobre Enseñanza y Divulgación de las Ciencias, 14(1), 199-214.

España, E. y Prieto, T. (2009) Educar para la sostenibilidad: el contexto de los problemas socio-científicos. Revista Eureka sobre Enseñanza y Divulgación de las Ciencias, 6, 345-354.

Evagorou, M., Jiménez-Aleixandre, M. P., y Osborne, J. (2012) "Should We Kill the Grey Squirrels?" A Study Exploring Students' Justifications and Decision-Making. International Journal of Science Education, 34(3), 401-428.

Feinstein N. (2011) Salvaging Science Literacy. Science Education, 95, 168 - 185.

Gresch, H., Hasselhorn, M. y Bögelholz, S. (2013) Training in Decision-making Strategies: An approach to enhance students' competence to deal with socio-scientific issues. International Journal of Science Education, 35(15), 2587-2607.

Heath, P. A., White, A. L., Berlin, D. F., y Park, J. C. (1987) Decision making: Influence of features and presentation mode upon generation of alternatives. Journal of Research in Science Teaching, 24(9), 821-833.

Jiménez-Aleixandre, M. P., y Pereiro Muñoz, C. (2002) Knowledge producers or knowledge consumers? Argumentation and decision making about environmental management. International Journal of Science Education, 24(11), 1171-1190.

Kifshe, R. (2012) Nature of Science and Decision Making. International Journal of Science Education, 34(1), 67-100.

Kolstø, S. D. (2001) Scientific Literacy for Citizenship: Tools for Dealing with the Science Dimension of Controversial Socioscientific Issues. Science Education, 85(3), 291-310. 
Kolstø, S. D., Bungum, B., Arnesen, E., Isnes, A.,Kristensen, T., Mathiassen, K., Mestad, I., Quale, A., Tonning, V., y Ulvik, M. (2006) Science students' critical examination of scientific information related to socioscientific issues. Science Education, 90, 632-655.

Kortland, K. (1996) An STS case study about students' decision making on the waste issue. Science Education, 80(6), 673-689.

Kuhn, D. (1991) The skills of argument. Cambridge, England: Cambridge University Press.

Lederman, N. G., y Lederman, J. S. (2014) Research on Teaching and Learning of Nature of Science. En N. G. Lederman y S. K. Abell (Eds.). Handbook of Research on Science Education, volumen II (pp.600-620). New York: Routledge.

Lee, Y. L., y Grace, M. (2012) Students' Reasoning and Decision Making About a Socioscientific Issue: A Cross-Context Comparison. Science Education, 96, 787-807.

Levinson, R. (2006) Towards a theoretical framework for teaching controversial socioscientific issues. International Journal of Science Education, 28, 1201-1224.

National Research Council (NRC). (1996) National science education standards. Washington, DC: National Academy Press.

Organización para la Cooperación y el Desarrollo Económico (OCDE). (2006) Assessing scientific, reading and mathematical literacy: A framework for PISA 2006. Paris: OCDE.

Organización para la Cooperación y el Desarrollo Económico (OCDE). (2013) PISA 2015 draft science framework. http://www.oecd.org/

Papadouris, N. (2012) Optimization as a Reasoning Strategy for Dealing With Socioscientific Decision-Making Situations. Science Education, 96, 600-630.

Porlán, R. y Martín del Pozo, R. (2004) The conceptions of inservice and prospective primary school teachers about the teaching and learning of science. Journal of Science Teacher Education, 15(1), 39-62.

Pedrinaci, E., Caamaño, A., Cañal, P. y De Pro, A. (2012) 11 ideas clave. El desarrollo de la competencia científica. Barcelona: Graó.

de Pro Bueno, A. J. (2013) Enseñar procedimientos: por qué y para qué. Alambique: Didáctica de las ciencias experimentales, (73), 69-76.

Ratcliffe, M. (1997) Pupil decision making about socio-scientific issues within the science curriculum. International Journal of Science Education, 19(2), 167-182.

Ratcliffe, M., y Grace, M. (2003) Science education for citizenship: Teaching socio-scientific issues. Philadelphia, PA: Open University Press.

Roberts, D. A. (2007) Scientific Literacy/ Science Literacy. En Abell, S. K. y Lederman, N. G. (Eds.). Handbook Research on Science Education. Mahwah, New Jersey: Lawrence Erlbaum.

Ryder, J. (2001). Identifying science understanding for functional scientific literacy. Studies in Science Education, 36, 1 - 44.

Sadler, T. D. (2004) Informal reasoning regarding socioscientific issues: A critical review of research. Journal of Research in Science Teaching, 41, 513-536

Sadler, T. D. (2009) Situated learning in science education: socio-scientific issues as contexts for practice. Studies in Science Education, 45(1), 1-42. 
Sadler, T. D. (2011) Socio-scientific Issues in the Classroom: Teaching, Learning and Research. Netherlands: Springer.

Sadler, T. D., Barab, S. A. y Scott, B. (2007) What do students gain by engaging in socioscientific inquiry?. Research in Science Education, 37(4), 371-391.

Sakschewski, M., Eggert, S., Schneider, S. y Bögeholz, S. (2014) Students Socioscientific Reasoning and Decision-making on Energy-related Issues-Development of a measurement instrument. International Journal of Science Education, 36 (14), 2291-2313.

Schreirer, M. (2012) Qualitative content analysis in practice. Londres: Sage.

Taber, K. S. (2017) Reflecting the Nature of Science in Science Education. En K. S. Taber y B. Akpan (Eds.). Science Education: an International Course Companion. (pp-23-37). The Netherlands: Sense Publishers.

Zeidler, D. L. (1997) The central role of fallacious thinking in science education. Science Education, 81(4), 483-496.

Zeidler, D. L., y Nichols, B. H. (2009) Socioscientific Issues: Theory and Practice. Journal of Elementary Science Education, 21(2), 49-58.

Zeidler, D. L., Applebaum, S. M. y Sadler, T. D. (2011) Enacting a socioscientific issues classroom: Transformative transformations. En T. D. Sadler (Ed.) Socio-scientific issues in science classrooms: Teaching, learning and research (pp. 277-306). Netherlands: Springer.

Zoller, U. (1982) Decision-making in future science and technology curricula. European Journal of Science Education, 4(1), 11-17. 